\title{
Mitochondrial K (ATP) Channels Opening: Pathways of Cytoprotection
}

\author{
Olga V Akopova* \\ Circulation department, Bogomoletz Institute of Physiology, Ukraine
}

Submission: July 13, 2017; Published: August 07, 2017

*Corresponding author: Olga V Akopova, Circulation department, Bogomoletz Institute of Physiology, NAS of Ukraine, Bogomoletz str. 4, 01601, Kiev, Ukraine, Tel: +38-044-256-24-96; Email: olga.akopova01@mail.ru

Abstract

Mitochondrial K (ATP) channels (mKATP channels) opening was shown to be cytoprotective under different pathophysiological conditions. Generally it was supposed that the modulation of ROS and RNS production could largely explain antiapoptotic and cytoprotective effects afforded by pharmacological mK(ATP) channels openers. But how to explain cytoprotection regardless of contradictory effects of mKATP channels opening on ROS production in mitochondria? Relying on published data, we try to answer this question there.

Keywords: Mitochondria; Mitochondrial K(ATP) channels; Reactive oxygen and nitrogen species; Cytoprotection

\section{Mini Review}

Mitochondrial ROS and RNS overproduction has a great cytotoxic potential well described in the literature. Several pathophysiological conditions and the action of cytotoxic agents are accompanied by excess ROS and RNS formation. These free radical products and their derivatives could easily modify cellular proteins, lipids, channels and enzymes and trigger multiple cell death pathways. Most dangerous is the interplay between ROS and RNS resulting in the formation of highly toxic products, such as $\bullet \mathrm{OH}$ radical and peroxynitrite. Their excess formation is known to severely aggravate pathophysiological conditions. mKATP channels openers were shown to be effective in prevention of apoptosis and necrosis under different pathological states, but, regardless of extensive studies of last two decades, pathways of their cytoprotective action are poorly understood. The progress is essentially hampered by still unknown molecular composition of mKATP channels. According to literary data, protection against cytotoxic agents, stress and pathophysiological conditions for the most part occurred via suppression of ROS and RNS overproduction. The effects of mKATP channels opening on RNS production are little studied yet, and most attention was focused on the regulation of ROS formation. However, main difficulty in explaining the mechanism(s) of cytoprotective action of mKATP channels openers this way is that mKATP channels opening was shown to differently affect ROS production. So, it worth mention to consider which effects on ROS production should be expected from mKATP channels opening.
Bioenergetic effects of mKATP channels opening. Generally, it is supposed that suppression of ROS overproduction by pharmacological mKATP channels openers (pinacidil, diazoxide, cromakalim, etc.) is based on bioenergetic effects of mKATP channel opening [1]. According to the present knowledge, ATPsensitive potassium transport, which is directed to the matrix space in energized mitochondria, dissipates $\Delta \mu \mathrm{H}$, a free energy generated by electron transport chain. Being energy consuming process, $\mathrm{K}+$ uptake always enhances state 4 oxygen consumption and the respiration rate. Unlike protonophoric uncoupling that diminishes $\Delta \mathrm{pH}$ component of $\Delta \mu \mathrm{H}$, uncoupling of the respiratory chain by mKATP channel opening results in elevated $\Delta \mathrm{pH}$ because of $\mathrm{K}+$ uptake into mitochondrial matrix. So, the dissipation of $\Delta \mu \mathrm{H}$ in the case of mKATP channel opening could largely occur at the cost of $\Delta \Psi \mathrm{m}$, and the effect of $\mathrm{K}+$ transport on $\Delta \Psi \mathrm{m}$ becomes important determinant of ROS production. The same is supposed to be true for RNS, because the dependence of RNS production on mitochondrial energy state too was shown in the literature.

In the heart and nervous system, mKATP channels were shown to be effective against a broad spectrum of cytotoxic agents. Protection of neurons by mKATP channels openers against glutamate [2], 1-methyl-4-phenylpyridinium (MPP (+)) [3], 3-nitropropionic acid [4] cytotoxicity as well as protection of cardiomyocytes against doxorubicin cytotoxicity [5] was shown to occur primarily via suppression of ROS and RNS production 
and the improvement of mitochondrial functional state. However, the main complexity in understanding physiological relevance of the modulation of ROS production by ATP-sensitive $\mathrm{K}+$ transport and cytoprotective effect thereof is that both elevation and suppression of ROS production ensuing from mKATP channel opening were shown to be protective under pathophysiological conditions [6-8]. The explanation could lay in high cell specificity of signaling pathways triggered by mKATP channels opening, however, published data on the pathways of cytoprotection afforded by mKATP channels opening are rather scarce.

Triggering of cytoprotective signaling Apart of simply bioenergetic effects, mKATP channels opening was shown to afford protection either by triggering protective signaling pathways or by the blockage of cell death pathways. For the most part, involvement of mKATP channels in cell signaling was shown to occur via modulation of ROS/RNS production, and one of most studied cellular targets of cytoprotection afforded by mKATP channels opening is mitochondrial permeability transition pore (mPTP), a giant nonselective mitochondrial channel involved in cell death pathways. As it was shown already in early works of Garlid's group, moderate elevation of ROS production by mKATP channels opening in cardiac mitochondria resulted in triggering one of the most effective pathways of cardioprotection that is the activation of mitochondrial protein kinase C (PKCE) with consequent blockage of MPTP [9].

The link between mKATP opening, the modulation of ROS production and the suppression of mitochondrial permeability transition pore (MPTP) activity resulting in a preconditioninglike effects is one firmly established in the literature $[6,10,11]$. Cytosolic stage of this pathway may include activation of phosphatydilinositol 3-kinase (PI3K), Akt, NO, cGMP and PKG pathway with resulting opening of mKATP channel, moderate increase in ROS production and activation of PKCE [6]. Rather surprising was that, different of the results obtained in the heart, in the brain protection mediated by PI3K-Akt-...-mKATP pathway with consequent neuronal preconditioning required suppression of ROS production [12].

Other signaling pathway such as suppression of cell death signaling [13] too was reported in the literature. Contradictory results with respect to ROS production only indicate that our understanding of protective pathways ensuing from mKATP opening is lacking of multiple links between bioenergetic consequences of mKATP channel opening and molecular mechanisms of protective signaling specific for different cell types.

\section{Conclusion}

Based on the published data, most effective cytoprotection by mKATP channels openers was afforded not so by simple modulation of the amount of ROS, RNS and their derivatives, but rather by triggering cell-specific protective mechanisms critically dependent on free radical formation. There is still a lack of knowledge on ROS signaling, but it seems that synergistic action of simply bioenergetic effects of mKATP channels opening and cell-specific signaling pathways triggered by ensuing modulation of ROS and RNS production play decisive role in cardio- and neuroprotective effects afforded by mKATP channels openers.

This synergistic linking between simply bioenergetic effects and protective signaling makes mKATP channels openers very effective pharmacological tool in cell and tissue protection against pathological states and broad variety of cytotoxicity agents. At present it seems that we are only at the beginning in understanding complex signaling network involved in antiapoptotic action of pharmacological mKATP channels openers. Disclosure of these mechanisms would help better understanding physiological role and functions of mKATP channels in a living organism.

\section{Reference}

1. Garlid KD, Paucek P (2003) Mitochondrial potassium transport: the $K$ (+) cycle. Biochim Biophys Acta 1606(1-3): 23-41.

2. Shukry M, Kamal T, Ali R, Farrag F, Almadaly E, et al. (2015) Pinacidil and levamisole prevent glutamate-induced death of hippo campal neuronal cells through reducing ROS production. Neurol Res 37(10): 916-23.

3. Xie J, Duan L, Qian X, Huang X, Ding J, et al. (2010) K(ATP) channel openers protect mesencephalic neurons against MPP+-induced cytotoxicity via inhibition of ROS production. J Neurosci Res 88(2): 428-437.

4. Gupta S, Sharma B (2014) Protective effects of phosphodiesterase-1 (PDE1) and ATP sensitive potassium (KATP) channel modulators against 3-nitropropionic acid induced behavioral and biochemical toxicities in experimental Huntington?s disease. Eur J Pharmacol 732: 111-122.

5. Lisa DH, Terje HL, Kjell OF, Fredrik L, Jan S (2014) Diazoxide protects against doxorubicin-induced cardiotoxicity in the rat. BMC Pharmacology and Toxicology 15: 28.

6. Garlid KD, Costa AD, Quinlan CL, Pierre SV, Dos SP (2009) Cardio protective signaling to mitochondria. J Mol Cell Cardiol 46(6): 858-866.

7. Qingxi F, Zhiqing S, Jinling Z, Naiyong G, Faying Qi, et al. (2013) Diazoxide preconditioning antagonizes cytotoxicity induced by epileptic seizures. Neural Regen Res 8(11): 1000-1006.

8. Lucas AM, Caldas FR, da Silva AP, Ventura MM, Leite IM, et al. (2016) Diazoxide prevents reactive oxygen species and mitochondrial damage, leading to anti-hypertrophic effects. Chem Biol Interact 261: 50-55.

9. Costa AD, Garlid KD (2008) Intra mitochondrial signaling: interactions among mitoKATP, PK Cepsilon, ROS, and MPT. Am J Physiol Heart Circ Physiol 295(2): H874-H882.

10. Busija DW, GasparT, Domoki F, Katakam PV, Bari F (2008) Mitochondrialmediated suppression of ROS production upon exposure of neurons to lethal stress: mitochondrial targeted preconditioning. Adv Drug Deliv Rev 60(13-14): 1471-1477.

11. Correia SC, Cardoso S, Santos RX, Carvalho C, Santos MS, et al. (2011) New insights into the mechanisms of mitochondrial preconditioningtriggered neuro protection. Current Pharmaceutical Design 17(31): 3381-3389. 
12. Gáspár T, Snipes JA, Busija AR, Kis B, Domoki F, et al. (2008) ROSindependent preconditioning in neurons via activation of mitoK(ATP) channels by BMS-191095. J Cereb Blood Flow Metab 28(6): 10901093.
13. Liang W, Chen M, Zheng D, Li J, Song M, et al. (2017) The opening of ATP-sensitive $\mathrm{K}+$ channels protects $\mathrm{H} 9 \mathrm{c} 2$ cardiac cells against the high glucose-induced injury and inflammation by inhibiting the ROS-TLR4necroptosis pathway. Cell Physiol Biochem 41: 1020-1034.

\section{Your next submission with Juniper Publishers} will reach you the below assets

- Quality Editorial service

- Swift Peer Review

- Reprints availability

- E-prints Service

- Manuscript Podcast for convenient understanding

- Global attainment for your research

- Manuscript accessibility in different formats ( Pdf, E-pub, Full Text, Audio)

- Unceasing customer service

Track the below URL for one-step submission https://juniperpublishers.com/online-submission.php 